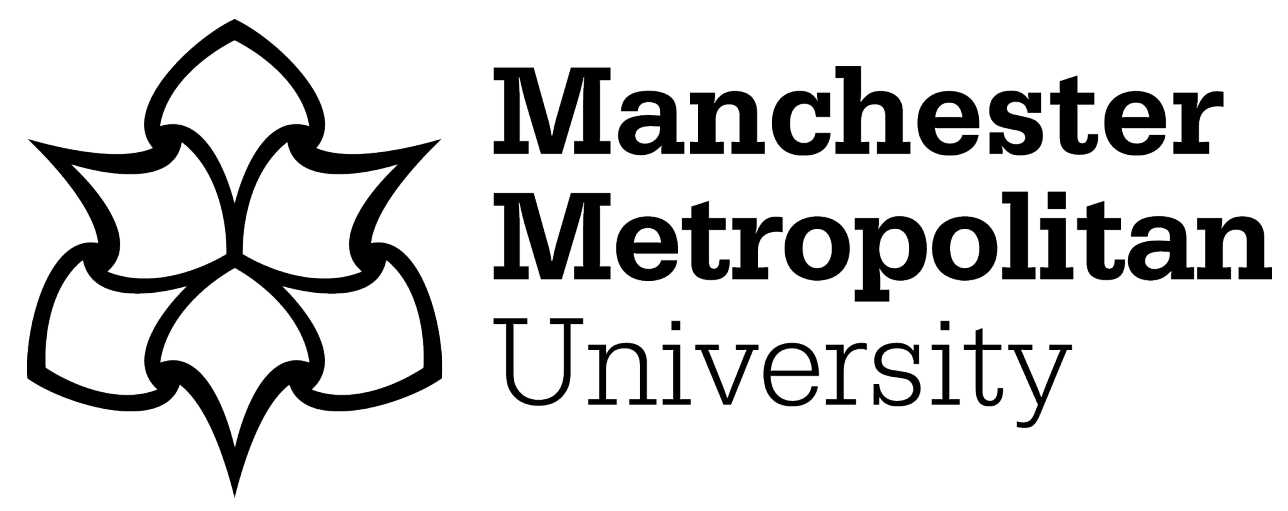

Grant, Robyn, Wong, Aimee, Fertan, Emre and Brown, Richard (2020) Whisker exploration behaviours in the 5xFAD mouse are affected by sex and retinal degeneration. Genes, Brain and Behavior, 19 (3). e12532. ISSN 1601-183X

Downloaded from: https://e-space.mmu.ac.uk/621751/

Version: Accepted Version

Publisher: Wiley

DOI: https://doi.org/10.1111/gbb.12532

Please cite the published version 


\title{
Whisker exploration behaviours in the 5xFAD mouse are affected by sex and retinal degeneration
}

\author{
Robyn A. Grant ${ }^{1}$ (1) | Aimee A. Wong ${ }^{2}$ | Emre Fertan ${ }^{2} \mid$ Richard E. Brown ${ }^{2}$
}

${ }^{1}$ Division of Biology and Conservation Ecology, Manchester Metropolitan University, Manchester, UK

${ }^{2}$ Department of Psychology and Neuroscience, Dalhousie University, Halifax, Nova Scotia,

Canada

Correspondence

Dr Robyn A. Grant, Division of Biology and Conservation Ecology, Manchester Metropolitan University, John Dalton Building, Chester Street, Manchester M1 5GD, UK.

Email: robyn.grant@mmu.ac.uk

\begin{abstract}
Active whisking in mice and rats is one of the fastest behaviours known in mammals and is used to guide complex behaviours such as exploration and navigation. During object contact, whisker movements are actively controlled and undergo robust changes in timing, speed and position. This study quantifies whisker movements in 6- to 7-month old male and female 5xFAD mice, and their C57/SJL F1 wild-type (WT) controls. As well as genotype, we examined sex differences and the effects of retinal degeneration ( $r d)$. Mice were filmed using a high-speed video camera at 500 frames per second (fps), under infrared light while behaving freely in three tasks: object exploration, sequential object exploration and tunnel running. Measures of whisker position, amplitude, speed and asymmetry were extracted and analysed for each task. The 5xFAD mice had significantly altered whisker angular positions, amplitude and asymmetry during object contacts and female 5xFAD mice with rd had lower mean angular positions during object contact. There were no significant effects of genotype on sequential object exploration or on tunnel running but differences due to sex and rd were found in both tasks, with female mice making larger and faster whisker movements overall, and mice with rd making larger and faster whisker movements during object contact. There were sex differences in whisker movements during sequential object exploration and females with rd had higher whisker retraction speeds in tunnel running. These data show that measuring whisker movements can quantify genotype and sex differences and the effects of rd during exploratory behaviour in these mice.
\end{abstract}

\section{KEYWORDS}

Alzheimer Disease, exploratory behaviour, mice, motor control, rodent models, sex differences, transgenic, vibrissae, whisker movements

\section{1 | INTRODUCTION}

Alzheimer Disease (AD) is an age-related progressive neurodegenerative disorder and is the most frequent form of dementia in elderly persons. ${ }^{1-4}$ Mouse models are key to understanding the progression of $A D$ and for developing new $A D$ treatments. ${ }^{5-8}$ Many of these mouse models develop $A \beta$-plaque pathology and cognitive dysfunction, but much less is known about their development of age-related sensorimotor deficits as occurs in AD. ${ }^{9}$ The majority of AD mouse models take over 6-months to develop phenotypic symptoms, but the transgenic 5xFAD mouse model exhibits amyloid plaques before 2 months of age ${ }^{10}$ and recapitulates many pathological changes observed in $A D$, including cognitive impairment, ${ }^{10,11}$ neuronal cell loss ${ }^{12}$ and reduced cerebral glucose uptake. ${ }^{13}$

Behaviourally, the 5xFAD mice have memory impairments, showed by a lack of recognition for novel objects at 6 to 7 months of age $^{14}$ and spatial memory deficits. ${ }^{15,16}$ They also have motor impairments after 9 months of age, ${ }^{17-19}$ age-related deafness ${ }^{20}$ and social impairments ${ }^{11}$ from 12 months of age. The 5xFAD mice also show a reduction of inhibitory interneurons in Layer IV of the whisker barrel cortex, which leads to changes in vibrissae-related behaviour that include a lack of whisker barbering in the home cage and an avoidance of enclosed spaces, which dissipates when the whiskers are trimmed. ${ }^{11}$ Although Jawhar et al ${ }^{12}$ found reduced anxiety in $5 \times \mathrm{FAD}$ 
mice in the elevated plus maze (EPM), the results of Flanigan et $\mathrm{al}^{11}$ suggest that these results are not due to reduced anxiety but to the mice avoiding the closed arms due to over-sensitive vibrissae. ${ }^{11}$ In addition, some 5xFAD and wild-type (WT) mice have retinal degeneration ( $r d$ ) and are completely blind ${ }^{21,22}$ as a result of the rd gene $\left(\mathrm{Pde} 6 \mathrm{~b}^{\mathrm{RD} 1}\right.$ ) in the SJL/J background strain. ${ }^{21}$ These mice, therefore, have a loss of vision and rely more on whisker touch to guide exploration navigation, and other behaviours.

Laboratory rats and mice use their whiskers as their primary sense for exploring their surroundings and employ them in navigation, object exploration and social interactions. ${ }^{23,24}$ Whiskers are regularly studied as a model system for investigating fundamental principles of sensory processing. ${ }^{25-27}$ During exploration, the whiskers move forwards and backwards (termed protractions and retractions) in a motion called whisking, which can occur at rates of up to $25 \mathrm{~Hz}$ in mice. ${ }^{28}$ Detailed quantitative behavioural analyses have showed that rodents alter the timing, spacing and positioning of their whiskers to maximize sensory information. ${ }^{28-31}$ For example, when contacting an object, they reliably and robustly: (a) reduce the retraction speed of the whisk so that their whiskers spend more time in contact with the surface of the object $^{30}$; (b) reduce the amplitude of whisker movements to increase contact duration ${ }^{29,30}$; (c) increase the frequency of whisking to maximize the amount of sampling against the surface ${ }^{32}$ and (d) reduce whisker angular positions to prevent whiskers being forced forward into the surface and enable light touches. ${ }^{30,31}$ Following unilateral contacts, whisker asymmetry increases, as the side contralateral to the contact is positioned more forward to increase whisker contact, and the side ipsilateral to the contact is positioned more backwards to enable light touches against the surface. ${ }^{28,30,31}$ Focussing of the whisker field towards objects during contacts is thought to indicate the spatial attention of the rodent. ${ }^{33,34}$

As the whisker movements of rodents are precisely controlled, and high-speed video footage can be measured using custom-made tracking software, whisking has been proposed as a quantitative behavioural measure of motor control and exploration abilities. ${ }^{27,35}$ Indeed, deficits in whisker movements have been seen in mouse models of motor disorders, such as amyotrophic lateral sclerosis ${ }^{36}$ and Huntington disease, ${ }^{37}$ as well as in models of anxiety. ${ }^{35}$ Because whisker movements are linked to attention and exploration, whisking behaviour might be a suitable model for testing cognitive functions in Alzheimer's model mice. In particular, we might expect to see whisker position and movement differences between 5xFAD and WT mice in sequential object exploration and tunnel running tasks, as 5xFAD mice have behavioural deficits in similar tasks. ${ }^{14}$

This study will, for the first time, assess the differences in whisker movements during exploratory behaviour between 5xFAD mice and their WT controls. Three behavioural tasks: object exploration (a welldefined task in whisker exploration), sequential object exploration (which is similar to the novel object task that is often used in $A D$ mouse models) and tunnel running (as used by Fragkouli et $\mathrm{al}^{14}$ to indicate sensitive whiskers in 5xFAD mice) will be used to determine if whisker movements differ in 5xFAD and WT mice at 6 to 7 months of age. As well as genotype differences, we will examine sex differences and the effect of rd on whisker movements in 5xFAD and WT mice in these three tasks.

\section{2 | METHODS}

\section{1 | Animals}

Fifty-nine 6- to 7-month old male and female 5xFAD mice were included in this study. All mice were the offspring of male hemizygous C57BL/6J $\times \mathrm{SJL} / J \quad F 2 \quad 5 \times F A D \quad$ (B6SJL-Tg (APPSwFILon, PSEN1*M146L*L286V) 6799Vas/Mmjax) and female WT C57BL/ 6J $\times$ SJL/J F1 mice obtained from Jackson Laboratories (Bar Harbour, Maine) and bred in our laboratory. The 5xFAD mice have five mutations; three on the APP gene, the Swedish (K670N/M671L), Florida (I716V) and London (V717I) mutations, and two mutations to the PSEN1 gene (PS1), which encodes presenilin-1PS1 (M146L and L286V). ${ }^{10}$ As a result of the rd gene $\left(\mathrm{Pde}^{\mathrm{RD}} \mathrm{b}^{\mathrm{RD}}\right)$ in the $\mathrm{SJL} / \mathrm{J}$ background strain, some mice have $r d$ and are blind. ${ }^{21}$ This type of $r d$ is early onset and severe ${ }^{21}$; indeed, due to early degeneration of the retina there is a complete loss of rods by 35 days of age. ${ }^{22}$ At 14 weeks of age, mice with the same RD1 mutation perform only at chance levels on a visual detection task, indicating that they were functionally blind. ${ }^{38}$ There may be some variation of symptoms during development due to interactions with other genes, ${ }^{39}$ however, by 6 months of age we would expect the mice to be completely blind, with no rods present. All mice were sexed and genotyped for the APP and PS1 transgenes, and the phosphodiesterase-6b retinal degeneration-1 (Pde6b ${ }^{\text {RD1}}$ ) allele by Dr Chris Sinal (Pharmacology Department, Dalhousie University) from tissue samples obtained from ear punches at weaning. In our colony, around $50 \%$ of pups born had the $5 x F A D$ gene and $25 \%$ had the Pde $6 b^{R D 1}$ gene.

The mice were weaned at 21 days of age, separated into groups of 2 to 4 same-sex littermates and housed in $30 \times 18 \times 12 \mathrm{~cm}$ polycarbonate cages with wire tops and ad lib access to food (Purina Rodent laboratory chow \#5001). The colony room was maintained at $22^{\circ} \mathrm{C} \pm 2^{\circ} \mathrm{C}$ with a $12: 12$ hour reversed light:dark cycle (lights off at 9:45 AM). All behavioural testing was completed during the dark (active) portion of the light:dark cycle. Mice were treated in accordance with the regulations set forth by the Canadian Council on Animal Care and the experimental protocol was approved by the Dalhousie University Committee on Animal Care.

\section{2 | Apparatus and tracking}

Mice were taken to the testing room, removed individually from their home cage and placed in a transparent, Perspex, rectangular arena $(30 \times 50 \times 15 \mathrm{~cm})$, which was lit from below by a bright, infrared light box (PHLOX LEDW-BL-400/200-SLLUB-Q-1R-24V). Mice were tested in three tasks that were designed to investigate whisker exploratory behaviours, including object exploration, sequential object exploration and tunnel running (Figure 1). Each mouse was introduced to the task arena, and clips were collected immediately during the initial exploration phase. The mouse spent only 10 to 12 minutes in each task arena, which was enough time to collect the allocated number of video clips for each task for each mouse as described below. In the object exploration task, a Pyrex bottle-stop was placed in the centre of the arena and a video clip was taken every time the mouse approached and investigated the object. Approximately 15 video clips 


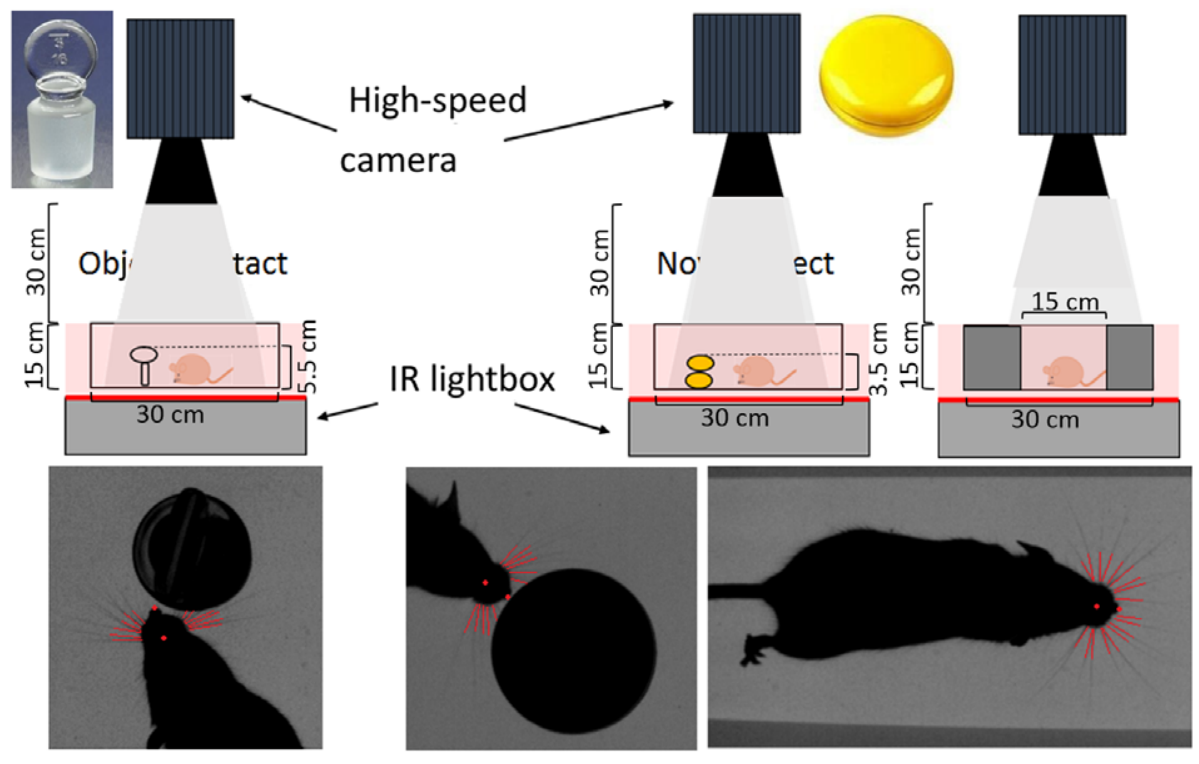

FIGURE 1 Schematic of the whisker behaviour tasks and tracking. The object exploration task (A) records exploration of a glass bottle stopper; the sequential object exploration task (B) records exploration of the bottle stopper and a novel small yo-yo object (photographs of both are inset). The tunnel running task $(C)$ involves the mouse travelling continually down an enclosed corridor. Behaviours were recorded in semi-darkness in all tasks using an infrared lightbox and a high-speed video camera, with points on the nose, head and whiskers (in red) tracked in all suitable videos

were collected from each mouse. The bottle-stop object $(5.5 \mathrm{~cm}$ tall) was semi-transparent, with a brushed textured bottom $(1.5 \mathrm{~cm}$ tall with a $2.3 \mathrm{~cm}$ diameter), the top was domed with a smooth, transparent, circle on top ( $3 \mathrm{~cm}$ tall with a $2.5 \mathrm{~cm}$ circle diameter), as can be seen in Figure $1 \mathrm{~A}$. In the sequential object exploration task, the same arena was used as for object exploration, with four clips collected when the mouse explored the original object (bottle-stop). This was then replaced with a second (novel) object, a mini yo-yo, and another five video clips were collected. The yo-yo object was $3.5 \mathrm{~cm}$ tall with a $3.5 \mathrm{~cm}$ diameter; it was yellow, with smooth, gloss plastic, as shown in Figure $1 \mathrm{~B}$. This is a modification of the usual novel object task, where the familiar and novel object are both included in the same trial, because the high-speed camera can only image the whiskers during object contact if it is directly above the object. If two objects were present, they would obscure the whiskers, as a vertical view cannot be achieved on both objects simultaneously. In the tunnel running task, black Perspex dividers were placed in the arena to make an enclosed tunnel of 40 long $\times 15$ wide $\times 15$ high $\mathrm{cm}$. Mice were filmed running up and down the tunnel and approximately eight clips were collected per mouse to represent one run along the tunnel. The sample size for each group is presented in the figure captions corresponding to each task (Figures 3, 5 and 7). All mice were filmed in the object exploration task first, then 1 to 2 days later in the tunnel running task, and then 3 to 4 days later in the sequential object exploration task.

In all tasks, filming was conducted under infrared light so the mouse would be filmed in perceived darkness. Mice were filmed from above using a digital high-speed video camera (Phantom Miro ex2) recording at $500 \mathrm{fps}$ with a shutter-speed of 1 millisecond and a resolution of $640 \times 480$ pixels, suspended $30 \mathrm{~cm}$ above the top of the arena (Figure 1). Multiple 700-millisecond video clips were collected opportunistically (by manual trigger) when the animal moved in the camera's field of view. This was in accordance with other mouse whisker and locomotion studies, ${ }^{35-37,40}$ as the camera was unable to store continuous footage, due to the large file size of high resolution, high-speed videos. Clips were selected with respect to a set of exclusion criteria (see individual sections for details), and in each clip, the snout and whiskers of the mice were tracked using the BIOTACT Whisker Tracking Tool. ${ }^{41}$ This tracker semi-automatically locates the orientation and position of the snout, and the angular position (relative to the midline of the head) of each identified whisker field. Tracking was validated by manually inspecting the tracking annotations overlaid on to the video frames (Figure 1).

The movement of the entire whisker field was determined, frameby-frame from the unsmoothed mean of all the tracked whisker angular positions for each side of the snout, to determine the naïve mean angle (nma), ${ }^{28}$ and the following variables were calculated from the whisker angular position data. Mean angular position was calculated per-clip, and averaged for the left and right whisker sides. Asymmetry was calculated as the mean difference between the left and right mean angular positions. The mean angular position was removed from the whisking angle time series and the root mean square (RMS) value was computed to give the RMS whisking amplitude. These time series were approximately sinusoidal, so the "peak-to-peak whisking amplitude" was estimated by multiplying the RMS whisking amplitude by $2 \sqrt{ } 2 .^{42}$ This estimate of amplitude is reasonably robust to accommodate departures from a purely sinusoidal pattern. Mean angular retraction and protraction speeds were calculated as the average velocity of all the backward (negative) and forward (positive) whisker movements, respectively. Angular position, amplitude, retraction and protraction velocities were calculated individually for each whisker side, and then averaged over the left and right sides to give one value of each per clip. Raw data from each task can be referred to in an excel document in Supporting Information Appendix S1. The time taken to explore each object (first and second) was recorded, however, this was limited 
to the length of the video clip (700 milliseconds), so could only include very short explorations. It did not differ between mice, over time or between the original and second object, so this data was not included in any analyses. Whisking frequency was also calculated using Fourier transforms of the whisker angular position traces, however, it did not differ significantly in any of the tests and was not included in any analyses. During behavioural testing, the genotype, gender and rd were recorded for each mouse, however, all clip selection, tracking and analysis was performed blinded. Although not directly measured here, we did not notice any stress or anxiety-like behaviours in any of the mice. Once introduced to the arena, they all explored away from the walls, at the centre of the arena, within the first minute, and performed a grooming bout at around 5 minutes into the testing session. There was no excessive defecation or urination by any of the mice.

\section{3 | Object exploration}

All 59 mice were used in this task (genotype: 30 5xFAD, 29 WT; sex: 30 male, 29 female; RD: 29 without, 30 with; exact mouse numbers for each group are shown in Figure 3), and a total of 662 video clips were collected from mice exploring the bottle-stop object. These clips were manually reviewed and selected for tracking using an automated tracker if: (a) the mouse was clearly in frame; (b) both sides of the face were visible; (c) the head was level with the floor (no extreme pitch or yaw); (d) there was a period prior to contact (pre-contact) of at least 50 frames and (e) a period during contact, where the nose-tip was clearly visible for at least 50 frames. All clips were then trimmed to include only periods of correct tracking, or removed from the analysis entirely. This left 387 clips for analysis, with 5 to 11 clips per mouse. Based on these criteria, around $44 \%$ of all clips were included for further analysis (genotype: 39\% 5xFAD, 48\% WT; sex: 48\% male, 39\% female; RD: $51 \%$ without, $36 \%$ with) (Appendix S1). Female 5xFAD mice with rd were the most affected $\left(\chi^{2}=16.0017, \mathrm{df}=11\right.$, $P=0.003)$. The inclusion criteria were relatively equal among all groups of mice, and the differences occurred in the automated whisker tracking stage, which we could not control.

A mixed-model multivariate analysis of variance (MANOVA) was conducted on all the whisking data with pre-contact and contact as the within factors, genotype (5xFAD, WT), sex (male, female) and rd (present, absent) as the between factors. Partial-eta squared $\left(\eta^{2} \mathrm{p}\right)$ was used for effect sizes (0.2 = small, $0.5=$ medium, $0.8=$ large). Individual MANOVAs using the same format were then conducted on just the male mouse data, and then the female mouse data, due to the large effects of sex multivariate ANOVAs were conducted to examine the effects of genotype and rd on the individual whisker variables. Analyses were also conducted to determine if there were differences in these whisker variables over subsequent contacts; however, no differences were observed in any of the mice so all contact data was combined for analysis.

\subsection{Sequential object exploration}

From the original 59 mice, 27 were randomly selected for this task (exact numbers for each group are shown in Figure 5), and a total of 282 video clips were collected from mice exploring the first object (bottle-stop) and then the second object (yo-yo). The clips were manually reviewed and selected for automatic tracking if they met the five criterion listed above for object exploration, which resulted in 108 clips for analysis, with 5 to 8 clips per mouse. Around 39\% of all clips were included for further analysis (genotype: 39\% 5xFAD, 41\% WT; sex: $43 \%$ male, $37 \%$ female; RD: $54 \%$ without, $43 \%$ with) based on these criteria, groups did not differ in the number of clips excluded $\left(\chi^{2}=0.9968, \mathrm{df}=11, P=0.910\right)$ (Appendix S1). A mixed-model MANOVA was conducted on all the contacting whisking data with the object type (original [first]: bottle-stop, and second: yo-yo) as the within factor, genotype (5xFAD, WT), sex (male and female), and rd (present, absent) as the between factors, using $\eta^{2} p$ for effect sizes. Individual MANOVAs were then conducted on the male mouse data, and then the female mouse data due to the large effect of gender; multivariate ANOVAs examined the effect of genotype and $r d$ on the individual whisker variables, Bonferroni corrections were not needed here and throughout (as per Bock ${ }^{43}$ ).

\section{5 | Tunnel running}

The same 27 mice from the sequential object exploration task were also used for this task (exact numbers for each group are shown in Figure 7), and a total of 227 clips were collected from mice in the tunnel running task. Clips were manually reviewed and selected for automatic tracking as described above, with the added criterion that the mouse travelled, without pausing, from one end of the tunnel to the other, resulting in 126 clips, with 4 to 6 per mouse. Around $58 \%$ of all clips were included for further analysis (genotype: 62\% 5xFAD, 55\% WT; sex: 58\% male, 59\% female; RD: $63 \%$ without, 54\% with) based on these criteria, which did not differ between groups $\left(\chi^{2}=0.8792\right.$, $\mathrm{df}=11, P=0.9275$ ) (Appendix S1). A multivariate ANOVA was conducted on all of the whisking data with genotype (5xFAD, WT), sex (male, female) and rd (present, absent) as the between factors. Individual multivariate ANOVAs were then conducted on male and female mouse data separately for genotype and rd effects.

\section{3 | RESULTS}

\section{1 | Object exploration}

During contact with the object, the whiskers were positioned less forward and more asymmetrically (Compare Figures 2a-d with Figures $2 \mathrm{e}-\mathrm{f}$ ), compared with whisker positions prior to contact. Overall, there were significant effects of genotype (MANOVA: $F_{6,373}=$ 2.275, $P=0.036, \eta^{2} \mathrm{p}=0.035$ ), sex (MANOVA: $F_{6,373}=10.172$, $P<0.001, \eta^{2} p=0.141$ ) and $r d$ (MANOVA: $F_{6,373}=3.194, P=0.005$, $\eta^{2} p=0.049$ ) on the whisker variables during contact with the object in this test. All mice made robust changes in whisker protraction and retraction speeds, angular positions, asymmetry and amplitude following contact with the object (Figure 3). All of the mice changed their whisker movements following contact with the object (MANOVA: $F_{6,375}=156.074, P<0.001$ ), with protraction speed (ANOVA: $F_{1,380}=414.615, P<0.001$, Figure 3a), retraction speed (ANOVA: $F_{1,380}=268.661, P<0.001$, Figure $3 \mathrm{~b}$ ) and amplitude 

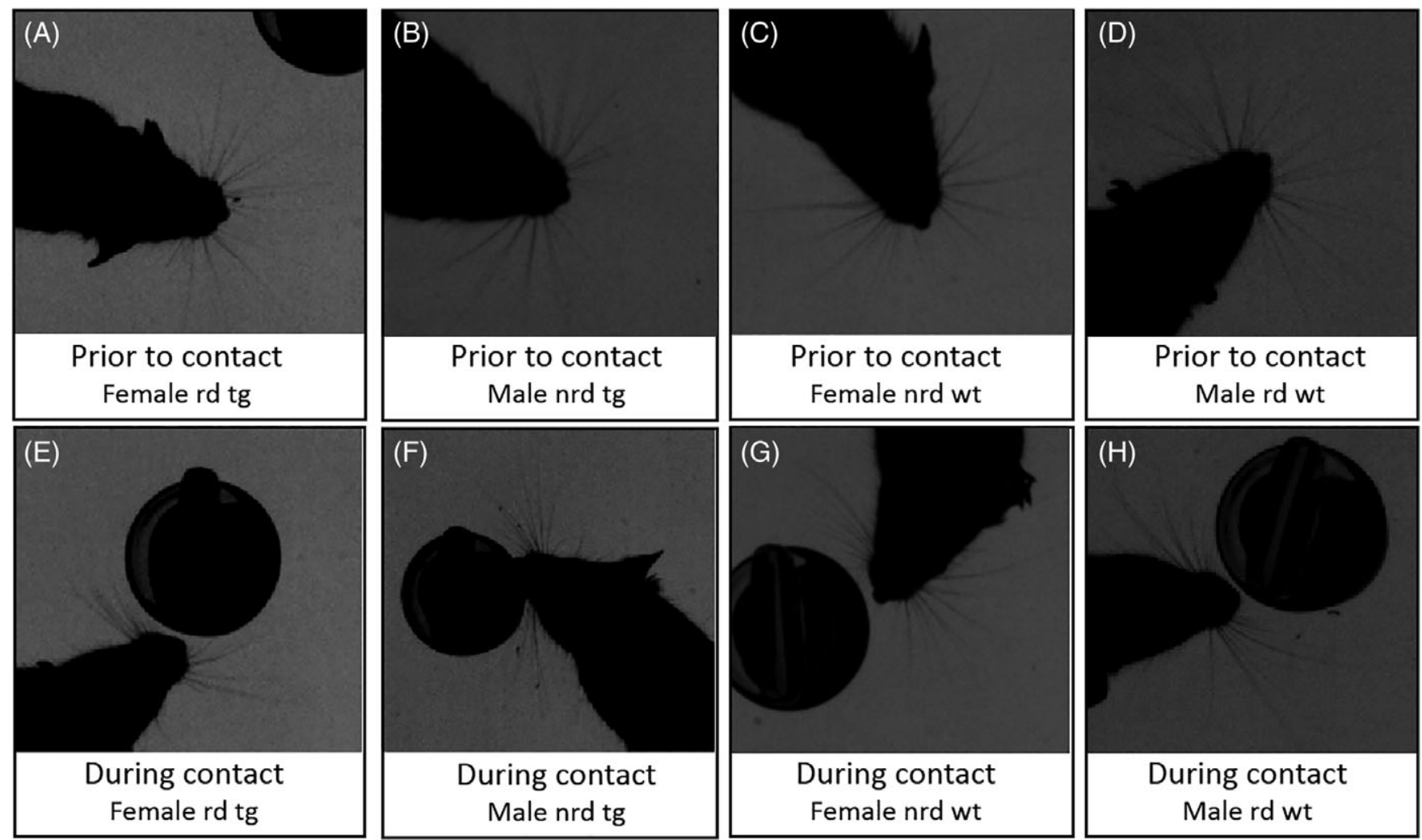

FIGURE 2 Screenshots of whisker positions prior to and during object contact. All screenshots were taken at maximum whisker protraction. Prior to contact, the whiskers were more forward and symmetrical $(A-D)$, than during contact $(E-H)$. NRD, no retinal degeneration, tg $=5 x F A D$ transgenic

(ANOVA: $F_{1,380}=747.503, P<0.001$, Figure 3e) all increasing following a contact. Mean angular position decreased following contact with the object (ANOVA: $F_{1,380}=188.300, P<0.001$, Figure 3c). Whisker asymmetry increased following a contact (ANOVA: $F_{1,380}=689.015, P<0.001$, Figure $3 d$ ) as can be seen by comparing Figure $2 \mathrm{a}-\mathrm{d}$ to Figure $2 \mathrm{e}-\mathrm{h}$.

As the difference between the sexes had such a large effect $\left(\eta^{2} p>0.8\right)$, with females having much larger and significant differences between pre-contact and contact values for protraction speed, retraction speed, mean angular position and asymmetry, and smaller differences in amplitude between pre-contact and during contact (Figure 3), data were then analysed separately for males and females. Male mice all showed robust changes in whisker variables following object contact and there was a significant genotype effect (MANOVA: $\left.F_{6,209}=2.841, P=0.011\right)$ but no significant effect of $r d$ on the whisker variables in male mice (MANOVA: $F_{6,209}=1.093, P=0.368$ ), but. Figure $3 a$ shows that male $5 x F A D$ mice had higher protraction speeds than WT males (ANOVA: $F_{1,214}=4.176, P=0.042$ ) and Figure $3 d$ shows that asymmetry was also higher in the $5 x F A D$ than the WT males (ANOVA: $F_{1,214}=15.306, P<0.001$ ). Indeed, the 5xFAD mice without $r d$ had the highest levels of whisker asymmetry, especially following object contact (ANOVA: $F_{1,214}=3.971, P=0.048$ ), which can be seen clearly by comparing Figure $2 f$ with Figure $2 \mathrm{e}, \mathrm{g}$,h.

Female mice also showed robust changes in whisker variables following object contact, with significant differences due to genotype (MANOVA: $F_{6,159}=3.473, P=0.003$ ) and $\mathrm{rd}$ (MANOVA: $\left.F_{6,159}=5.734, P<0.001\right)$. Female 5xFAD mice had significantly lower mean angular position values than WT females (ANOVA: $F_{1,164}=6.677, P=0.011$, Figure 3c). Females with $\mathrm{rd}$ had higher values of mean angular position than females without $r d$ before object contact, but had lower mean angular position scores following contact (ANOVA: $F_{1,164}=17.468, P<0.001$; Figure $3 c$ ), as can be seen in Figure 2a,c,e,f. Female 5xFAD mice with rd had the lowest mean angular position scores following a contact (ANOVA: $F_{1,164}=5.782$, $P=0.017$; Figure $3 c$ ). Females with rd also had higher values of asymmetry (ANOVA: $F_{1,164}=9.162, P=0.003$; Figure $3 d$ ) and amplitude than females without rd, following object contact (ANOVA: $F_{1,164}=13.309, P<0.001$; Figure 3e).

\section{2 | Sequential object exploration}

All mice showed significant changes in whisker movements on the second object, compared with the original object, with all mice having consistently smaller amplitudes on the second object. Overall, there were no significant effects of genotype (MANOVA: $F_{5,40}=2.056$, $\left.P=0.091, \eta^{2} p=0.204\right)$, or rd (MANOVA: $F_{5,40}=1.719, P=0.152$, $\eta^{2} p=0.177$ ) on the whisker variables exploring the original and second object. However, there was a large and significant sex difference (MANOVA: $F_{5,40}=4.442, P=0.003, \eta^{2} p=0.357$ ), so data was then analysed separately for males and females.

Figure 4 shows whisker traces of a male $5 \times$ FAD mouse without rd, which illustrates the decrease in whisker amplitude and asymmetry when investigating the second object compared with the original object, which was observed in all mice, irrespective of genotype, sex 

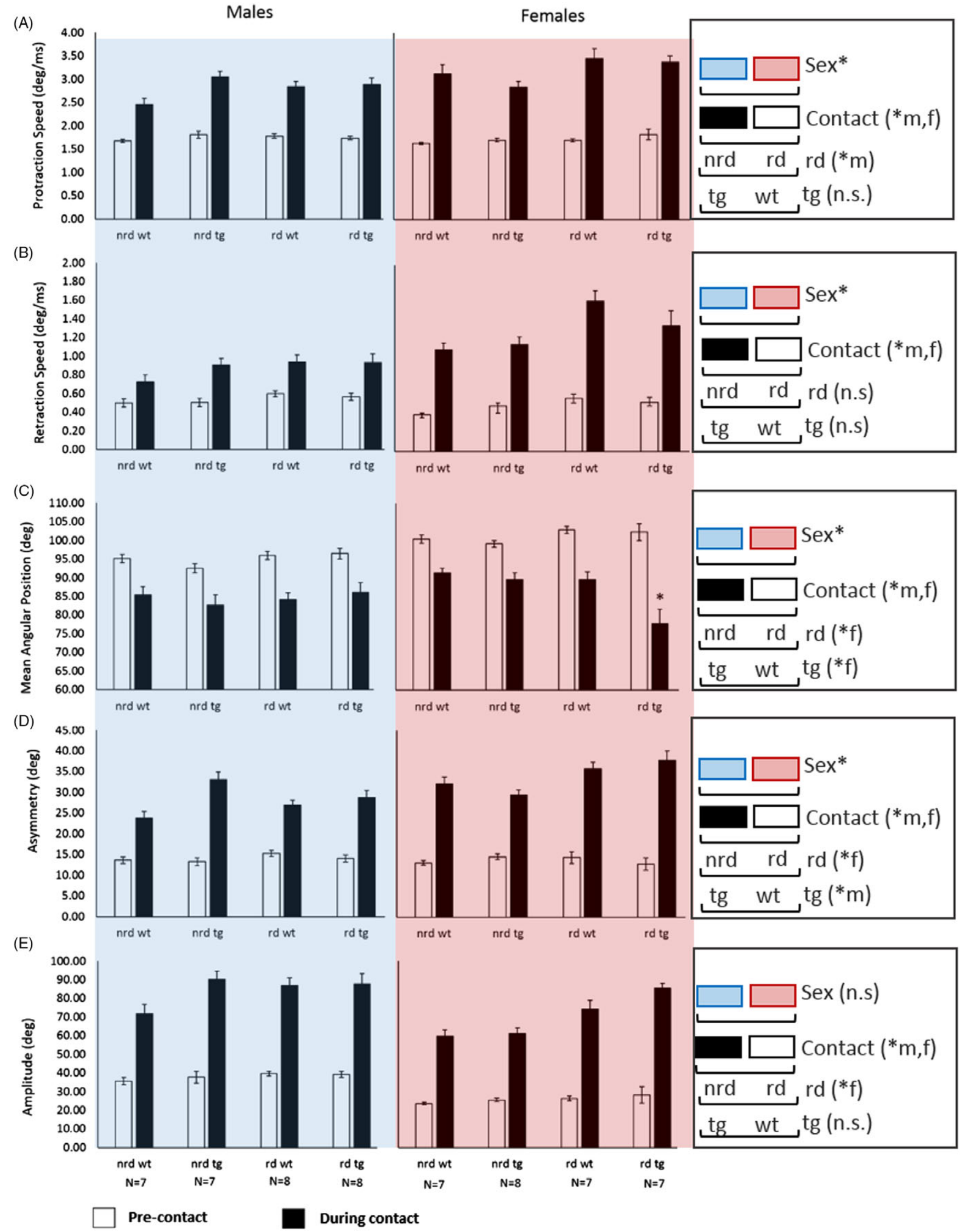

FIGURE 3 Summary of whisker movements during the pre-contact and object contact periods in male (left) and female (right) mice. Mean ( \pm SEM) protraction speed (A) and retraction speed (B) both increased during object contact. Angular position (C) decreased during contact, while asymmetry (D) and amplitude (E) both increased during object contact. Female 5xFAD mice with retinal degeneration had lower angular positions during contact than other mice. $* P<0.05$, with the sex of the interaction $(m=$ male and $f=$ female) indicated in the key. NRD, no retinal degeneration, tg $=5 x F A D$ transgenic. All whisker variables measured showed significant changes on object contact (indicated by the white and black bars). The column on the right summarizes the results of the ANOVAs for differences due to sex, object contact, retinal degeneration and genotype 
and $r d$. Both male (ANOVA: $F_{1,26}=6.976, P=0.014$; Figure $5 d$ ) and female mice (ANOVA: $F_{1,19}=19.612, P<0.001$; Figure $5 d$ ) showed significant decreases in whisker amplitude while exploring the second object. Male mice also showed a decrease in asymmetry during exploration of the second object (ANOVA: $F_{1,26}=9.289, P=0.005$; Figure $5 c$ ) and male mice with rd had greater asymmetry when investigating the original object, and lower asymmetry with the second object than males without rd (ANOVA: $F_{1,26}=4.253, P=0.049$; Figure 5c).

Female mice showed a reduction in protraction speed (ANOVA: $F_{1,19}=4.916, P=0.039$; Figure $5 \mathrm{a}$ ) and retraction speed (ANOVA: $F_{1,19}=30.379, P<0.001$; Figure $5 b$ ) during second object exploration, compared with exploration of the original object. Female 5xFAD mice had greater retraction speeds with the original object, and lower retraction speeds with the second object, than WT females (ANOVA: $F_{1,19}=7.958, P=0.013$; Figure $5 b$ ). Females with $r d$ had greater retraction speeds with the original object and lower retraction speeds with the second object, than females without rd (ANOVA: $F_{1,19}=5.926, P=0.025$; Figure $5 b$ ) Indeed, 5xFAD female mice with rd had the highest retraction speeds on the original object $(P=0.032$; Figure 4d) and the lowest in the second object.

\section{3 | Tunnel running}

Overall, there was no significant effect of genotype (MANOVA: $\left.F_{5,114}=1.633, P=0.157, \eta^{2} p=0.067\right)$, rd (MANOVA: $F_{5,114}=1.512$ $P=0.192, \eta^{2} p=0.062$ ) or sex (MANOVA: $F_{5,114}=2.298, P=0.050$, $\eta^{2} p=0.092$ ) on the whisker variables during the tunnel running task. The data was split by sex to further investigate genotype and rd effects during tunnel running. Examples of whisker traces from female WT mice show that those with rd had higher amplitudes and retraction speeds than those without rd (Figure 6). Female mice showed no significant genotype differences on these measures $(P>0.05)$, but females with $\mathrm{rd}$ showed significantly higher retraction speeds (ANOVA: $F_{1,64}=5.041, P=0.028$; Figure $7 a$ ) and amplitudes than

(A)

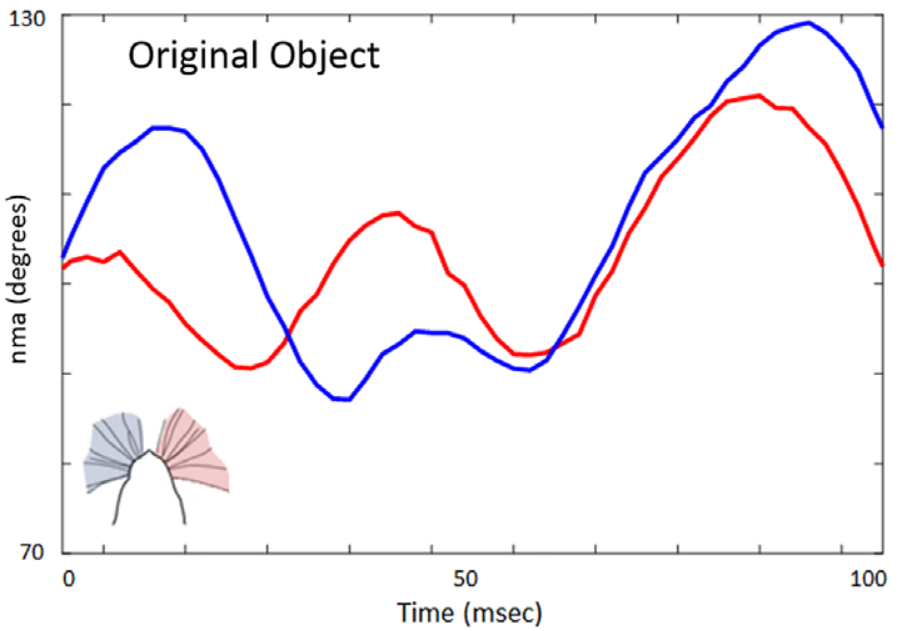

females without rd (ANOVA: $F_{1,64}=4.401, P=0.040$; Figure $7 b$ ). Male mice showed no significant effects of genotype or rd on their whisker retraction speed or amplitude (all $P>0.05$, Figure 7a,b), and traces looked very similar to those in Figure $6 \mathrm{c}$.

\section{4 | DISCUSSION}

All mice made robust changes in whisker movements and positions when they contacted an object. The 5xFAD mice differed from WT mice in whisker movements during object exploration, and mice with rd differed from sighted mice in whisker positions and movements in the object exploration, sequential object exploration and tunnel running tasks. The sex of the mice had the largest effects on whisker movements, with male and female mice having large and significant difference in all three of the experimental tasks.

All mice robustly altered their whisker movements during object exploration by increasing protraction and retraction speeds, asymmetry and amplitude and decreasing their angular positions (Figure 3). An increase in contact-induced asymmetry is associated with orienting to an object and maximizing whisker contacts. ${ }^{31,34}$ The decrease in angular position is common during exploration; as a mouse orients towards an object, the amount the whiskers are moved forward is reduced to enable light touches, with the whiskers not striking too hard onto the surface. ${ }^{30,31}$ The robustness of these findings across all mice supports the use of measures of whisker movements to quantify object exploration in rodents. Exploration has been associated with lower whisker retraction speeds ${ }^{30,36}$ and amplitudes, ${ }^{29,30}$ so these increases in whisker retraction speed and amplitude contradict results from previous studies. However, they do indicate an increase in the scanning area of the whiskers. Perhaps these variables are more task-specific or variable than asymmetry and angular position.

We did not observe any significant differences between 5xFAD and WT mice in whisker movements or other general behaviour in the tunnel running task. Flanigan et $\mathrm{al}^{11}$ found that 5 xFAD mice avoided a

(B)

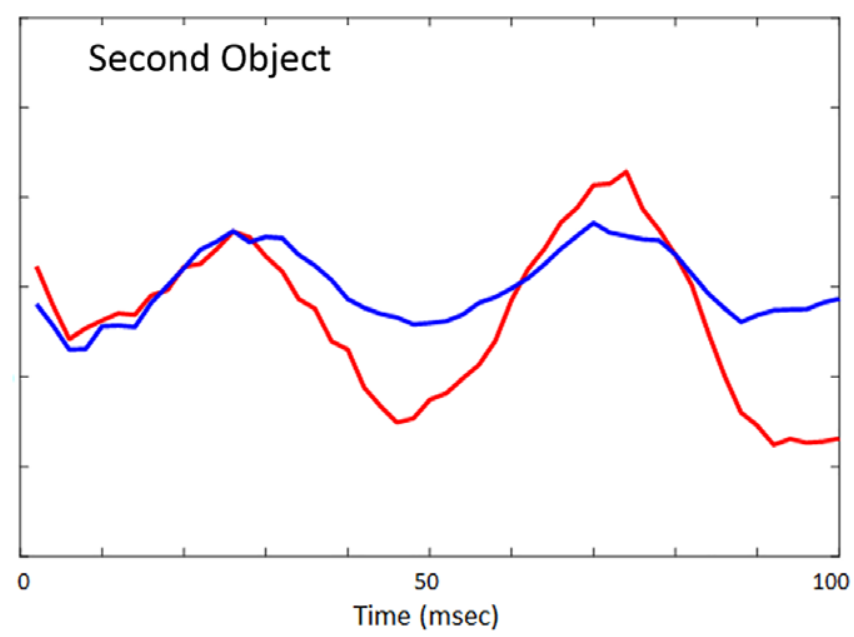

FIGURE 4 Examples of whisker traces from the sequential object exploration task. Whisker measurements of a male 5xFAD mouse without retinal degeneration exploring the original object $(\mathrm{A})$; and the second object $(\mathrm{B})$. Whisker amplitude and asymmetry were both larger when investigating the original than the second object. Left whiskers are shown with blue lines, and right whiskers with red lines. nma is the naïve mean angle, the average angular positions of all the whiskers on that side of the face 
closed arm tunnel; however, when their whiskers were removed they no longer avoided the tunnel, suggesting that there might be an increased sensitivity, or aversive overstimulation, of their vibrissae. Although we did not observe any evidence of this in our studies, it may account for the reduced angular position values during contact in the 5xFAD female mice during the object exploration task (Figure 2c). Reduced angular positions would ensure lighter touches and lower force whisker contacts. ${ }^{31}$ Although no previous study has measured whisker movements in 5xFAD mice, aspects of their exploratory behaviour have been examined. In an open field test, 12 month-old $5 x F A D$ mice spent more time away from the walls of the arena than WT mice, indicating a reduction in their anxiety. ${ }^{12}$ In our object exploration test, the object was in the centre of an open field and we did not notice the 5xFAD mice approaching it faster, or spending more
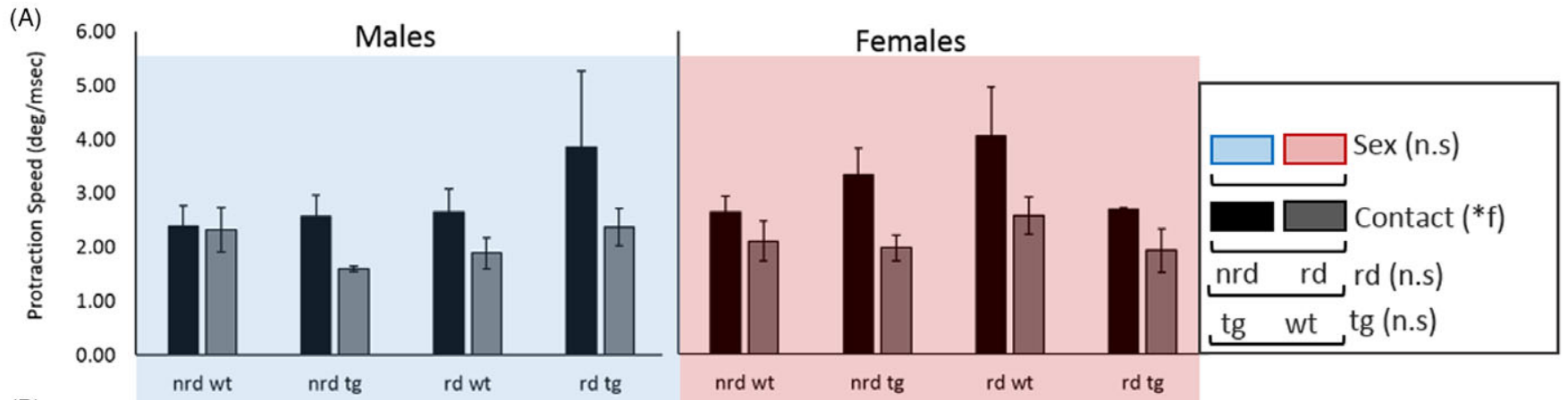

(B)
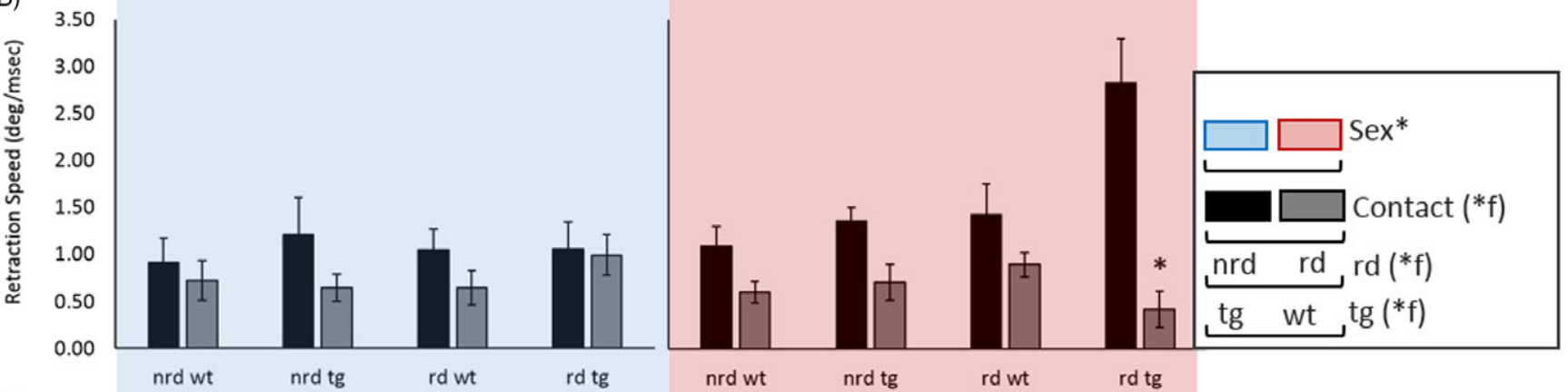

(C)

(C)

(D)

$-5.00$
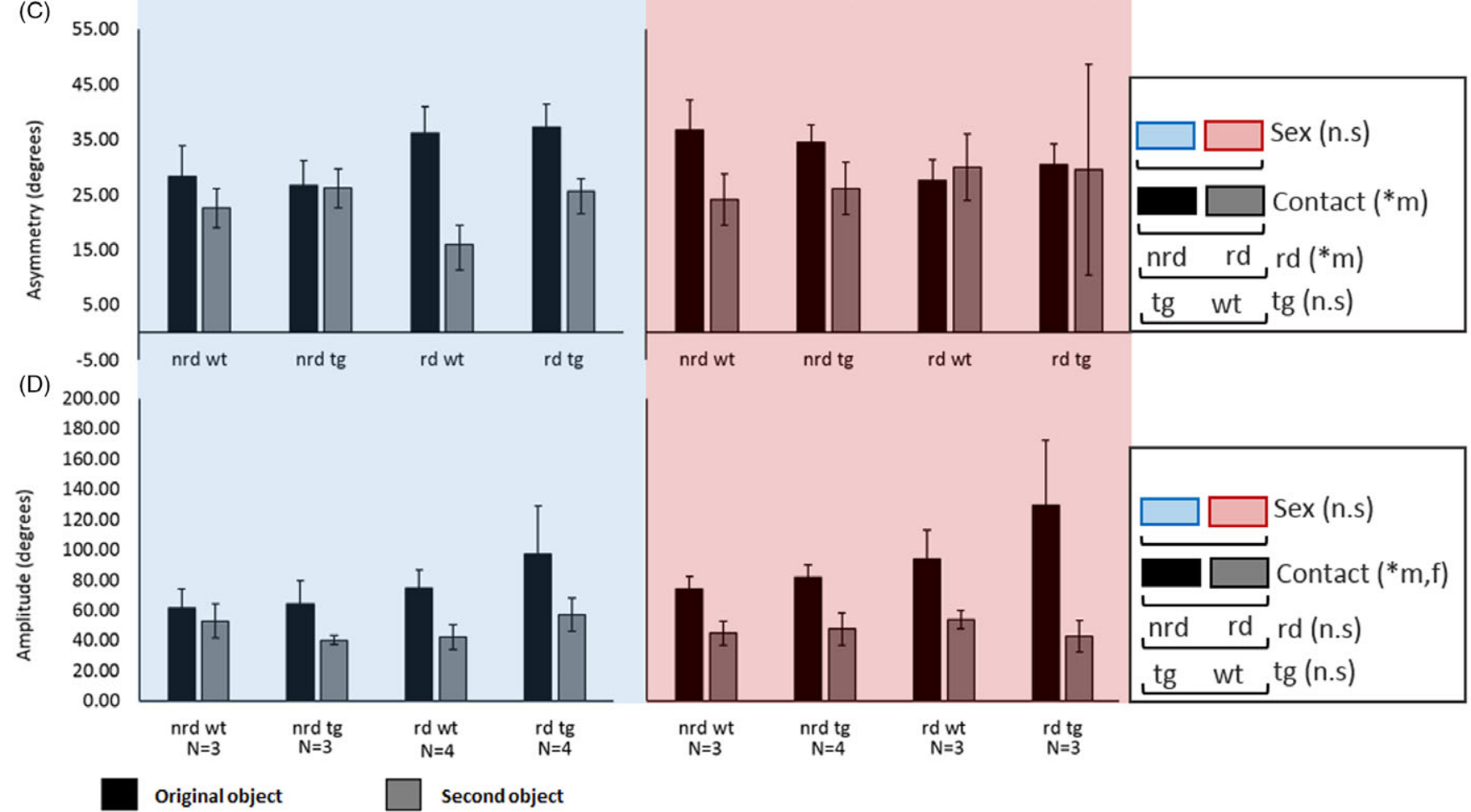

FIGURE 5 Summary of the whisker movements when exploring the original object and the second object by male (left) and female (right) mice. Mean ( \pm SEM) whisker protraction speed $(A)$; retraction speed $(B)$; asymmetry $(C)$ and amplitude $(D)$. Female $5 x F A D$ mice with retinal degeneration had the highest retraction speed on the original object, and lowest retraction speed on the second object, with the sex of the interaction ( $m=$ male and $f=$ female) indicated in the key NRD, no retinal degeneration; tg, 5xFAD transgenic. The column on the right indicates whether there are significant differences due to sex, during contact on the original and the second object $(* P<0.05)$ 
time in the centre of the arena than WT mice, perhaps because our mice were younger. The 6- to 7-month-old 5xFAD mice have been reported to spend less time exploring novel objects ${ }^{14}$; however, we did not observe a genotype difference in the time exploring the original or second (novel) object. In fact, the reduction in whisker retraction speed in the 5xFAD female mice with rd during exploration of the second object is more representative of an increase in object exploration duration. Indeed, reducing retraction speeds is thought to increase the time the whiskers spend in contact with an object, ${ }^{30}$ increasing the exploratory time overall.

In many instances, the 5xFAD mice showed an overall increase in object exploration using their whiskers. However, these changes differed between tasks and sexes. In the object exploration task, female 5xFAD mice had lower angular position values (Figure 2c; Figure 3c) and male 5 XFAD mice had increased asymmetry during object contact (Figure 2b; Figure 3d). In the sequential object task, female 5xFAD mice with rd had the lowest retraction speeds (Figure $5 b$ ). These behaviours are all associated with an increase in controlled and focussed exploration of an object. ${ }^{30,33}$ No motor phenotype has previously been documented in 5xFAD mice at 6 to 7 months of age, ${ }^{17-19}$ therefore, these differences in exploratory whisker movements may be due to cognitive or emotional (ie, anxiety) differences between the transgenic and WT mice. However, although not directly measured here, we did not notice any stress or anxiety-like behaviours in any of the mice. Therefore, if both motor deficits and anxiety-like behaviour are unlikely in the 5xFAD mice, changes in whisker behaviour may be due to alterations in sensory processing. It has been suggested that 5xFAD mice might have hypersensitive whiskers due to a reduction of inhibition in the barrel cortex, ${ }^{11}$ with the barrel cortex producing large amplitude neural responses with a broad spatial spread following a whisker deflection. ${ }^{44}$ It may be that whisker signals are not processed efficiently in the cortex, therefore, their exploratory movements increase to gain more sensory information; or that they are over-sensitive, so decrease whisker angular positions in order to reduce the force applied to the whisker from an object contact. Further investigations into whisker object contacts and forces in 5xFAD mice might help to tease apart these hypotheses (ie, Campagner et $\mathrm{al}^{45}$ ).

There were significant effects of rd on how the mice moved their whiskers; however, these changes were not consistent between different tasks and showed sex differences. Female mice with rd showed an increase in asymmetry and decrease in angular position in the object exploration task, and male mice with rd showed an increase in asymmetry in the sequential object exploration task (Figure 5), consistent with an increase in exploration. However, mice with rd also showed an increase in amplitude, in females in the object exploration task (Figure 3) and tunnel running task (Figure 7), and males in the

(A)

(B)

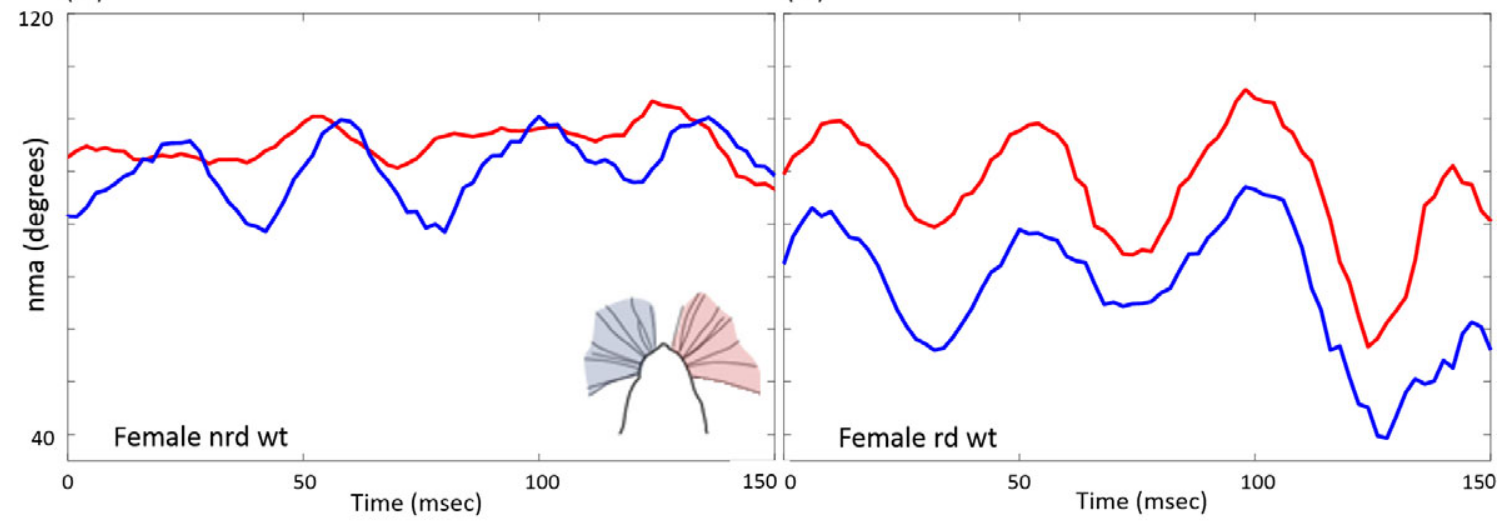

(C)

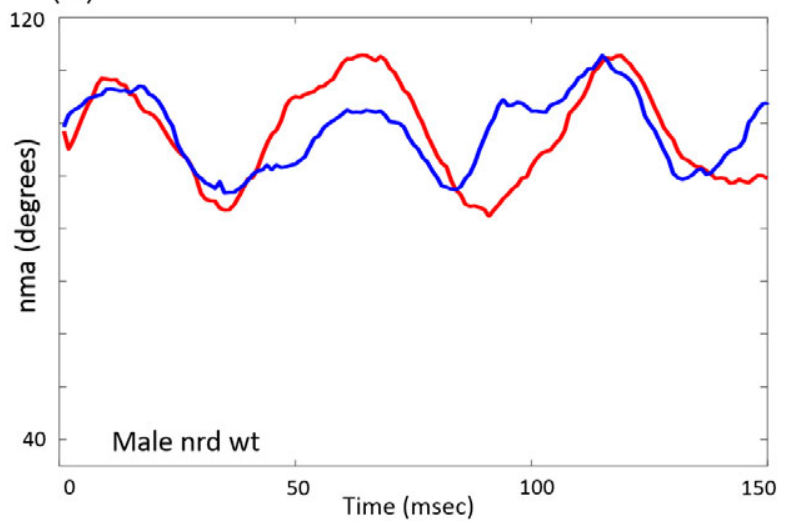

FIGURE 6 Example of whisker traces of three wild-type mice during the tunnel running task. Wild-type females with retinal degeneration (B) had larger whisker amplitudes than WT females with no retinal degeneration (A). They also had faster retraction speeds, indicated by the steep slopes of the retractions. Wild-type male mice had relatively small whisker amplitudes (C), similar to (B), with no differences between those with and without retinal degeneration. Left whiskers are shown with blue lines, and right whiskers with red lines. nma is the naïve mean angle, the average angular positions of all the whiskers on that side of the face 
sequential object exploration task (Figure 5). Arkley et $\mathrm{al}^{33}$ found that rats with retinal dystrophy, when introduced to an unfamiliar environment, also had larger amplitudes, compared with sighted animals. It might be that without sight, blind rodents will increase the area that they are scanning over (increasing amplitude) to prevent collisions. There were also many instances where the mice with rd did not show an increase in exploratory behaviour, for instance, female mice with rd showed an increase in retraction speed in the sequential object exploration task and male mice with rd showed an increase in protraction speed during the object exploration task. It is likely, therefore, that the interaction of vision and whisker touch might be quite complex. New strains of 5xFAD mice do not carry the rd allele Pde $6 b^{R D 1}$, however, the amyloid phenotype is less robust in these animals (jax.org 2017). We know that mice with rd are impaired in visuospatial learning tasks, but not in non-visual tasks, ${ }^{46}$ so tactile sensory information from the whiskers may help compensate for lack of visual information.

Both sexes showed robust changes in all whisker position and movement variables following contact in the object exploration task, and in amplitude in the sequential object exploration task but there were no sex differences in each task. Female 5xFAD mice have a higher density of plaques than males at a similar age, ${ }^{17}$ but it is not clear from our data whether 5xFAD female mice are more affected in their whisker movements than the males (compare Figures 3, 5, 7). However, the sex differences observed in this study indicate that the 5xFAD female mice used their whiskers more than males to investigate objects in both the object exploration and the sequential object exploration tasks (Figures 3 and 5). No studies have yet explored sex differences in whisker movement in different strains of mice or rats and these needs to be completed before whisker measurements become a standard behavioural test for exploration and object recognition. There are significant sex differences in mouse models, in general, ${ }^{47,48}$ and in AD model mice specifically, in terms of disease pathology, ${ }^{14,17}$ lifespan and healthspan ${ }^{49,50}$ and behaviour. ${ }^{14}$

\section{1 | Conclusions}

Our study showed significant differences in genotype, sex and rd in whisker movements in 5xFAD and WT mice. The largest differences were found between male and female mice across the three tasks tested. Whisker movements also differed between mice with and without rd in all three tasks, and the object exploration and sequential object exploration tasks showed genotype differences in whisker movements. Therefore, the effects of sex and rd might interact with or confound aspects of mouse whisking behaviour more than the effects of the genetic mutations in the 5xFAD mice. Characterizing sex differences in whisker movements in other mouse models of $A D$ and in non-transgenic mice and rats would be important in understanding some of the differences that we observed. All mice showed robust and reliable changes in measures of whisker movements from pre-contact to contact on the object exploration task (Figure 3). Therefore, this is likely to be a reliable test for future studies measuring object exploration. The sequential object exploration task also showed promising results. While there were strong effects of sex in some of the whisker variables, the reduction of whisker amplitude in
(A)

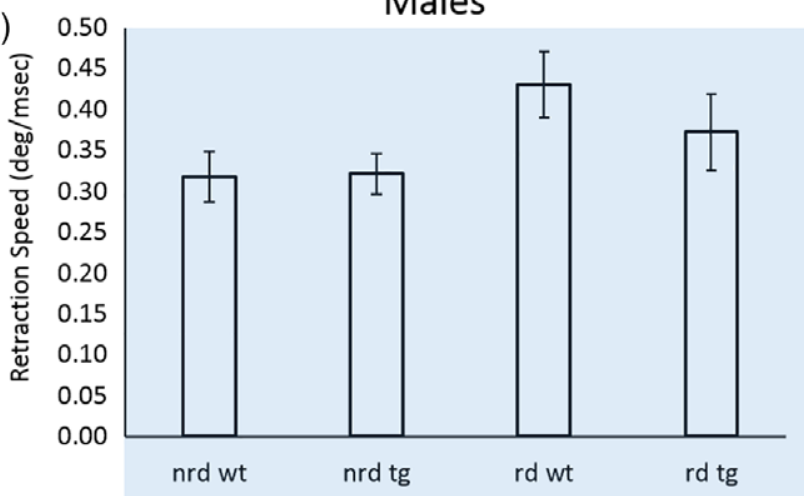

Males

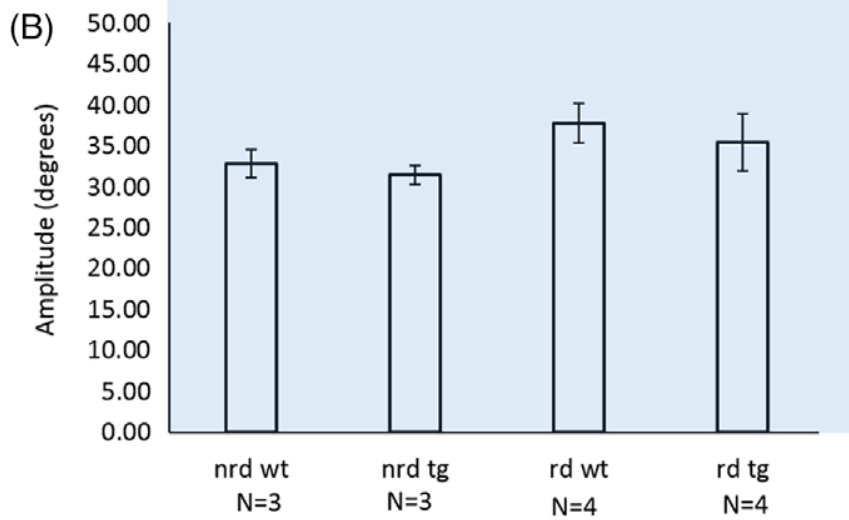

Females

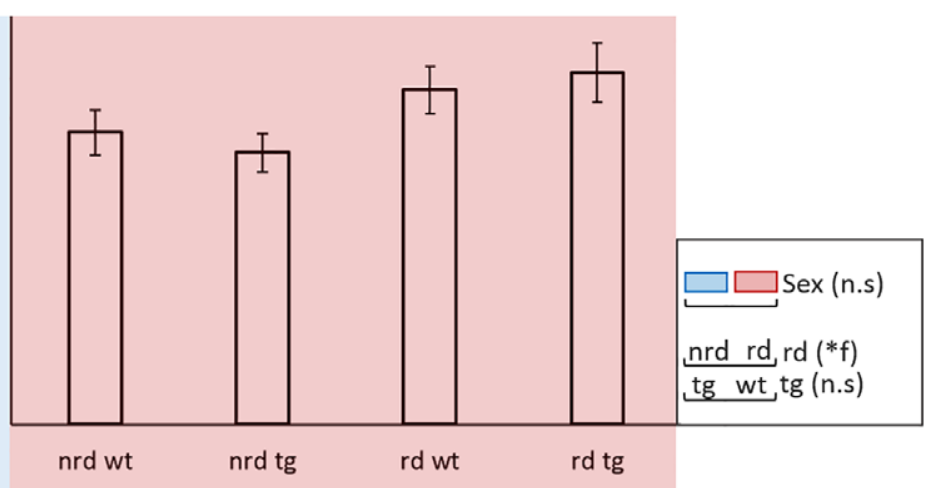

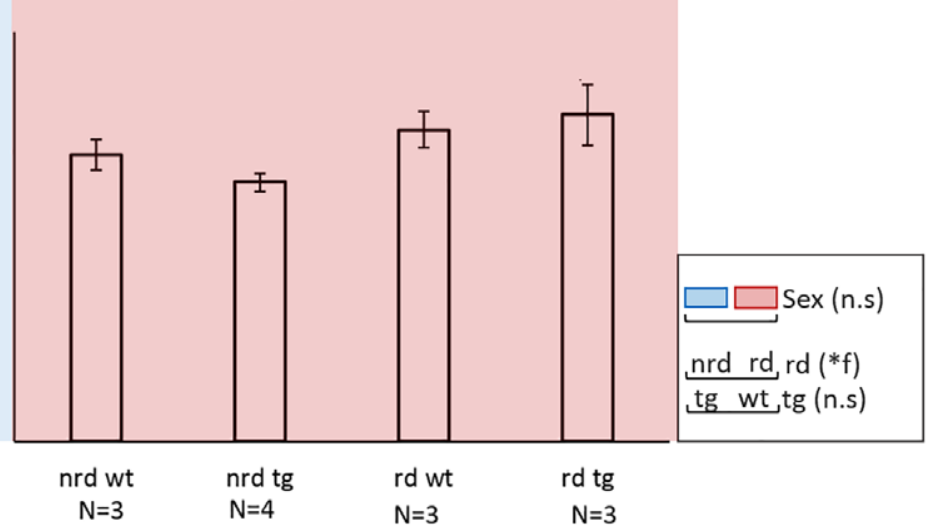

FIGURE 7 Summary of whisker movements of male (left) and female (right) mice during tunnel running. Mean ( \pm SEM) whisker retraction speed (A); and amplitude (B). $* P<0.05$, with the sex of the interaction ( $m=$ male and $f=$ female) indicated in the key: NRD, no retinal degeneration; tg: $\mathrm{Tg}=5 \mathrm{xFAD}$ transgenic 
all mice encountering the second (novel) object indicates a focussing of attention ${ }^{33,34}$ and suggests an increase in exploration of the new object, $^{29,30}$ as might be expected in habitution-dishabituation experiments. ${ }^{51-54}$ Usually, novel object tasks simply measure the duration of time spent exploring the new object, whereas measuring whisker movements quantifies the tactile aspects of this behaviour. Therefore, measuring whisker movements during object exploration tasks may give new insights into rodent behaviours, and their phenotypic changes in mouse models of disease. Finally, the careful analysis of whisking behaviour is essential for understanding the neural basis of tactile sensation and the ability of mice to "see" with their whiskers. ${ }^{55-57}$ Understanding the neural basis of whisking behaviour and its changes with genotype, sex, age and disease state may lead to new insights into the neural basis of behaviour. ${ }^{58,59}$

\section{ACKNOWLEDGMENTS}

We are extremely grateful to Shelina Lisle for tracking the tunnel running experiment. We would also like to thank the undergraduate students at Dalhousie University who helped with some of the video selection.

\section{ORCID}

Robyn A. Grant (D) https://orcid.org/0000-0002-3968-8370

\section{REFERENCES}

1. Jeong S. Molecular and cellular basis of neurodegeneration in Alzheimer's disease. Mol Cells. 2017;40:613-620.

2. Knopman DS, Haeberlein SB, Carrillo MC, et al. The National Institute on Aging and the Alzheimer's Association research framework for Alzheimer's disease: perspectives from the research roundtable. Alzheimers Dement. 2018;14:563-575.

3. Scheltens P, Blennow K, Breteler MM, et al. Alzheimer's disease. Lancet. 2016;388:505-517.

4. Vinters HV. Emerging concepts in Alzheimer's disease. Annu Rev Pathol. 2015;10:291-319.

5. Bilkei-Gorzo A. Genetic mouse models of brain ageing and Alzheimer's disease. Pharmacol Ther. 2014;142:244-257.

6. Jankowsky JL, Zheng H. Practical considerations for choosing a mouse model of Alzheimer's disease. Mol Neurodegener. 2017;12:89. https:// doi.org/10.1186/s13024-017-0231-7.

7. Puzzo D, Gulisano W, Palmeri A, Arancio O. Rodent models for Alzheimer's disease drug discovery. Expert Opin Drug Discovery. 2015;10(7): 703-711.

8. Webster SJ, Bachstetter AD, Nelson PT, Schmitt FA, Van Eldik LJ. Using mice to model Alzheimer's dementia: an overview of the clinical disease and the preclinical behavioural changes in 10 mouse models. Front Genet. 2014;5:88.

9. Albers MW, Gilmore GC, Kaye J, et al. At the interface of sensory and motor dysfunctions and Alzheimer's disease. Alzheimers Dement. 2014; 11:70-98.

10. Oakley $\mathrm{H}$, Cole SL, Logan $\mathrm{S}$, et al. Intraneuronal $\beta$-amyloid aggregates, neurodegeneration, and neuron loss in transgenic mice with five familial Alzheimer's disease mutations: potential factors in amyloid plaque formation. J Neurosci. 2006;26:10129-10140.

11. Flanigan TJ, Xue $Y$, Kishan Rao S, Dhanushkodi A, McDonald MP. Abnormal vibrissa-related behavior and loss of barrel field inhibitory neurons in 5xFAD transgenics. Genes Brain Behav. 2014;13:488-500.

12. Jawhar S, Trawicka A, Jenneckens C, Bayer TA, Wirths O. Motor deficits, neuron loss, and reduced anxiety coinciding with axonal degeneration and intraneuronal $A \beta$ aggregation in the 5XFAD mouse model of Alzheimer's disease. Neurobiol Aging. 2012;33:196.e29-196.e40.
13. Macdonald IR, DeBay DR, Reid GA, et al. Early detection of cerebra glucose uptake changes in the 5XFAD mouse. Curr Alzheimer Res. 2014;11:450-460.

14. Fragkouli A, Tsilibary EC, Tzinia AK. Neuroprotective role of MMP-9 overexpression in the brain of Alzheimer's 5xFAD mice. Neurobiol Dis. 2014;70:179-189.

15. Arendash GW, Gordon MN, Diamond DM, et al. Behavioral assessment of Alzheimer's transgenic mice following long-term $A \beta$ vaccination: task specificity and correlations between $A \beta$ deposition and spatial memory. DNA Cell Biol. 2001;20:737-744.

16. Urano T, Tohda C. Icariin improves memory impairment in Alzheimer's disease model mice (5xFAD) and attenuates amyloid $\beta$-induced neurite atrophy. Phytother Res. 2010;24:1658-1663.

17. Bhattacharya S, Haertel C, Maelicke A, Montag D. Galantamine slows down plaque formation and behavioral decline in the 5XFAD mouse model of Alzheimer's disease. PLoS One. 2014;9(2):e89454.

18. O'Leary TP, Mantolino HM, Stover K, Brown RE. Age-related deterioration of motor function in male and female 5xFAD mice from 3-16 months of age. Genes, Brain Behav. 2018a; Submitted.

19. O'Leary TP, Robertson A, Chipman PH, Rafuse VF, Brown RE. Motor function deficits in the 12 month-old female 5 XFAD mouse model of Alzheimer's disease. Behav Brain Res. 2018b;337:256-263.

20. O'Leary TP, Shin S, Fertan E, et al. Reduced acoustic startle response and peripheral hearing loss in the 5xFAD mouse model of Alzheimer's disease. Genes Brain Behav. 2017;16:554-563.

21. Chang B, Hawes NL, Hurd RE, Davisson MT, Nusinowitz S, Heckenlively JR. Retinal degeneration mutants in the mouse. Vis Res. 2002;42:517-525.

22. Dalke C, Graw J. Mouse mutants as models for congenital retinal disorders. Exp Eye Res. 2005;81(5):503-512.

23. Grant RA, Arkley KP. Matched filtering in active whisker touch. In: Von der Emde G, Warrant E, eds. The Ecology of Animal Senses. New York City, USA: Springer International Publishing; 2016:59-82.

24. Kleinfeld $D$, Deschênes $M$. Neuronal basis for object location in the vibrissa scanning sensorimotor system. Neuron. 2011;72:455-468.

25. Diamond ME, Von Heimendahl M, Knutsen PM, Kleinfeld D, Ahissar E. 'Where' and 'what' in the whisker sensorimotor system. Nat Rev Neurosci. 2008;9:601-612.

26. Kleinfeld D, Ahissar E, Diamond ME. Active sensation: insights from the rodent vibrissa sensorimotor system. Curr Opin Neurobiol. 2006; 16:435-444.

27. McElvain LE, Friedman B, Karten HJ, et al. Circuits in the rodent brainstem that control whisking in concert with other orofacial motor actions. Neuroscience. 2018;368:152-170.

28. Mitchinson B, Grant RA, Arkley K, Rankov V, Perkon I, Prescott TJ. Active vibrissal sensing in rodents and marsupials. Philos Trans $R$ Soc $B$. 2011;366:3037-3048.

29. Carvell GE, Simons DJ. Biometric analyses of vibrissal tactile discrimination in the rat. J Neurosci. 1990;10:2638-2648.

30. Grant RA, Mitchinson B, Fox CW, Prescott TJ. Active touch sensing in the rat: anticipatory and regulatory control of whisker movements during surface exploration. J Neurophysiol. 2009;101:862-874.

31. Mitchinson B, Martin CJ, Grant RA, Prescott TJ. Feedback control in active sensing: rat exploratory whisking is modulated by environmental contact. Proc R Soc Lond B Biol Sci. 2007;274:1035-1041.

32. Berg RW, Kleinfeld D. Rhythmic whisking by rat: retraction as well as protraction of the vibrissae is under active muscular control. J Neurophysiol. 2003;89:104-117.

33. Arkley K, Grant RA, Mitchinson B, Prescott TJ. Strategy change in vibrissal active sensing during rat locomotion. Curr Biol. 2014;24: 1507-1512.

34. Mitchinson B, Prescott TJ. Whisker movements reveal spatial attention: a unified computational model of active sensing control in the rat. PLoS Comput Biol. 2013;9(9):e1003236.

35. Grant RA, Cielen N, Maes K, et al. The effects of smoking on whisker movements: a quantitative measure of exploratory behaviour in rodents. Behav Process. 2016;128:17-23.

36. Grant RA, Sharp PS, Kennerley AJ, et al. Abnormalities in whisking behaviour are associated with lesions in brain stem nuclei in a mouse model of amyotrophic lateral sclerosis. Behav Brain Res. 2014;259: 274-283. 
37. Garland H, Wood NI, Skillings EA, Detloff PJ, Morton AJ, Grant RA. Characterisation of progressive motor deficits in whisker movements in R6/2, Q175 and Hdh knock-in mouse models of Huntington's disease. J Neurosci Methods. 2018;300:103-111.

38. Wong AA, Brown RE. Visual detection, pattern discrimination and visual acuity in 14 strains of mice. Genes Brain Behav. 2006;5: 389-403.

39. Hackam AS, Strom R, Liu D, et al. Identification of gene expression changes associated with the progression of retinal degeneration in the rd1 mouse. Invest Ophthalmol Vis Sci. 2004;45(9):2929-2942.

40. Hewitt BM, Yap MH, Hodson-Tole EF, Kennerley AJ, Sharp PS, Grant RA. A novel automated rodent tracker (ART), demonstrated in a mouse model of amyotrophic lateral sclerosis. J Neurosci Methods. 2018;300:147-156.

41. Perkon I, Košir A, Itskov PM, Tasič J, Diamond ME. Unsupervised quantification of whisking and head movement in freely moving rodents. J Neurophysiol. 2011;105:1950-1962.

42. Chatfield C. The Analysis of Time Series: An Introduction. 6th ed. London, England: Chapman and Hall; 2003.

43. Bock RD. Multivariate Statistical Methods in Behavioural Research New York, NY: Mc-Graw-Hill; 1975.

44. Maatuf $\mathrm{Y}$, Stern EA, Slovin H. Abnormal Population Responses in the Somatosensory Cortex of Alzheimer's Disease Model Mice. Scientific reports. 2016;6:24560.

45. Campagner D, Evans MH, Bale MR, Erskine A, Petersen RS. Prediction of primary somatosensory neuron activity during active tactile exploration. Elife. 2016;5:e10696.

46. Brown RE, Wong AA. The influence of visual ability on learning and memory performance in 13 strains of mice. Learn Mem. 2007;14(2): 134-144.

47. Jonasson Z. Meta-analysis of sex differences in rodent models of learning and memory: a review of behavioral and biological data. Neurosci Biobehav Rev. 2005;28:811-825.

48. Schellinck HM, Cyr DP, Brown RE. How many ways can mouse behavioral experiments go wrong? Confounding variables in mouse models of neurodegenerative diseases and how to control them. Adv Study Behav. 2010;41:255-366.

49. Kane AE, Shin $S$, Wong AA, et al. Sex differences in healthspan predict lifespan in the 3x Tg-AD mouse model of Alzheimer's disease. Front
Aging Neurosci. 2018;10:172. https://doi.org/10.3389/fnagi.2018. 00172.

50. Rae EA, Brown RE. The problem of genotype and sex differences in life expectancy in transgenic AD mice. Neurosci Biobehav Rev. 2015; 57:238-251.

51. Bolivar VJ. Intrasession and intersession habituation in mice: from inbred strain variability to linkage analysis. Neurobiol Learn Mem. 2009; 92:206-214.

52. Ennaceur A. One-trial object recognition in rats and mice: methodological and theoretical issues. Behav Process. 2010;89:232-238.

53. Heyser CJ, Chemero A. Novel object exploration in mice: not all objects are created equal. Behav Process. 2012;89:232-238.

54. Schellinck HM, Rooney E, Brown RE. Odors of individuality of germfree mice are not discriminated by rats in a habituation-dishabituation procedure. Physiol Behav. 1995;57:1005-1008.

55. Curtis JC, Kleinfeld D. Seeing what the mouse sees with its vibrissae: a matter of behavioral state. Neuron. 2006;50:524-526.

56. Diamond ME, Arabzadeh E. Whisker sensory system - from receptor to decision. Prog Neurobiol. 2013;103:28-40.

57. Sofroniew NJ, Svoboda K. Whisking. Curr Biol. 2015;25:R137-R140.

58. Ferezou I, Bolea S, Petersen CC. Visualizing the cortical representation of whisker touch: voltage-sensitive dye imaging in freely moving mice. Neuron. 2006;50:617-629.

59. Stüttgen MC, Schwarz C. Barrel cortex: what is it good for? Neuroscience. 2018;368:3-16.

\section{SUPPORTING INFORMATION}

Additional supporting information may be found online in the Supporting Information section at the end of the article.

How to cite this article: Grant RA, Wong AA, Fertan E, Brown RE. Whisker exploration behaviours in the 5xFAD mouse are affected by sex and retinal degeneration. Genes, Brain and Behavior. 2018;e12532. https://doi.org/10.1111/ gbb.12532 